\title{
Preparation of Activated Carbon from seeds residues of (Urtica dioica) plants
}

Osamah m. Majeed Taha
Khalid S. Tawfiq

Deparetment of Chemistry

Edication College of pure science

University of Mosul

osamahmohamed81@gmail.com khalid.tawfiq@gmail.com subhi.jarullah@gmail.com

Subhi M. Jarullah

DOI: $\underline{10.33899 / \text { edusj.2019.162970 }}$

Received

$26 / 09 / 2018$
Accepted

03 / 12 / 2018

\begin{abstract}
Activated Carbon characterized by special importance for it's large use in industry field and pollution struggle it has been prepared from different sources and ways.

In this study activated carbon was prepared from seed residues of (Urtica dioica) plants after extractions of vegetable to preparation the biodiesel.

The process of activated carbon preparation include the following steps:

1) Primary carbonization of the seed residues at $\left(300^{\circ} \pm 50^{\circ} \mathrm{c}\right)$.

2) Secondary carbonization of product results from the primary carbonization with exiss amount of sodium hydroxide $\{(1: 2)$ (seeds residues: $\mathrm{NaOH})\}$ at $\left(500 \pm 25^{\circ} \mathrm{c}\right)$ for $3 \mathrm{hrs}$. The product washed with: distillated water treated with $10 \%$ $\mathrm{HCl}$, washed with distillated water and ther dried at $110^{\circ} \mathrm{c}$ for $24 \mathrm{hrs}$.

Number of tests concerning the activated carbon were parformed including all types of activated carbon prepared in steps 1and 2, these tests including:

Physiochemical tests such as humidity, density, ash content and mechanical resistance and measurements of iodine number and methylene blue.

The studying results show that carbonization seed residues with sodium hydroxide in (2) give the best results of the prepared activated carbon than in (1).
\end{abstract}

Key words: (Activated Carbon, seed residues of (Urtica dioica) plants) 


\section{تحضير الفحم المنشط من مخلفات بذور نبات القربص (Urticadioica)}

صبحي محسن جار الله

خالا سعيد توفيق

أسامة محمد مجيا طه

$$
\text { قسم الكيمياء/ كلية التربية للعلوم الصرفة }
$$

subhi.jarullah@gmail.com khalid.tawfiq@gmail.com osamahmohamed81@gmail.com DOI: $10.33899 /$ edusj.2019.162970

$$
\text { القبول }
$$

2018 / 12 / 03

$$
2018 \text { / } 09 \text { / } 26
$$

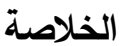

نظراً للأهمية الكبيرة للكاربون المنشط واستعماله في مدى واسع في الصناعة ومكافحة التلوث، فقد تم

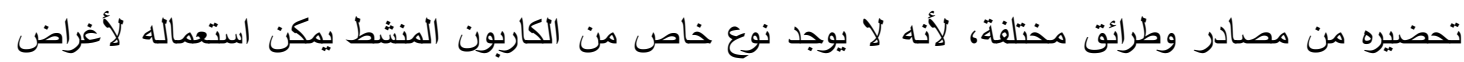
متعددة.

وفي هذه الدراسة تم تحضير الكاربون المنشط من مخلفات بذور نبات القريص بعد استخلاص الزيت منها لتحضير الديزل الحيوي. تمت عملية التحضير كالآتي:

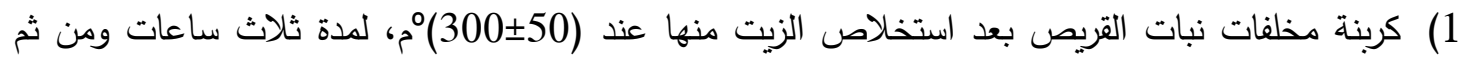

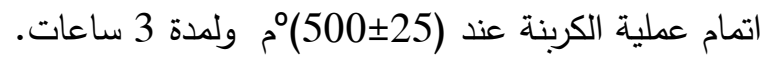

2) كربنة المخلفات مع زيادة من هيدروكسيد الصوديوم بنسبة (2:1) (مخلفات بذور : هيدروكسيد) عند 25+550 مُ لمدة ثلاث ساعات.

كلا المادتين الناتجتين من (1) و (2)، غسلت بالماء المقطر عدة مرات، ومن ثم عوملت مع محلول 10\% حامض الهيدروكلوريك، ثم غسلت بالماء المقطر لحين التعادل. تم تحديد خواص الكاربون المنشط الناتج عن طريق قياس قابلية الامتزاز كل من: محلول اليود وصبغة لهنة

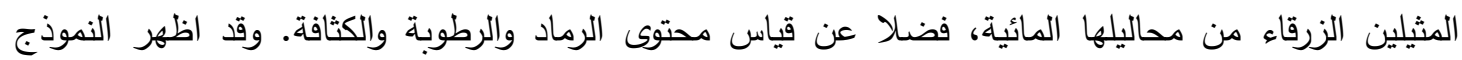
المحضر في الفقرة (2) نتائج افضل من النموذج المحضر في الفقرة (1). الكلمات المفتاحية: (الكاربون المنشط، مخلفات بذور نبات القريص)

\section{Introduction}

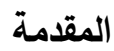

\section{Activated Carbon} الكاربون المنشط :- (المعنه

يعتبر الكاربون المنشط احد اكثر المواد المستعملة في الصناعة على نطاق واسع وذلك لانه من المواد التي لها مساحة سطحية كبيرة وتركيب مسامي متطور ولهذا السبب فان للكاربون المنشط سعة امتزاز عالية للمواد الكيميائية بانواعها المختلفة(1). هو عبارة عن مادة مسامية بهيئة متبلمرة تعاني خلال انتاجها او تحضيرها

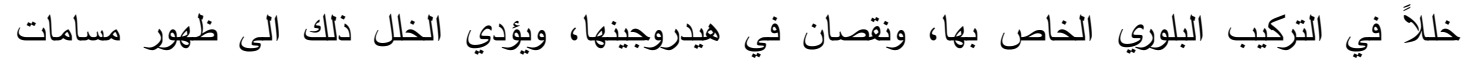
تكون غير مستقرة من ناحية المحتوى الطاقي او الفعالية. وتوجد هذه المسامات غالبا على السئل السطوح

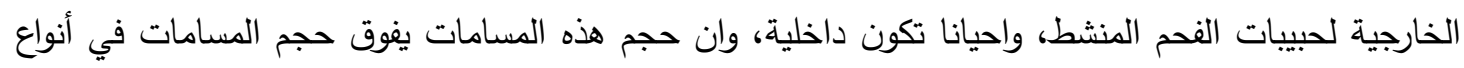
الفحم الاعتيادية، وبالتالي تكون قدرتها على الامتزاز أكبر (2). 
يمثل الكاربون المنشط احد صور الكاربون غير المتبلورة، اذ له القابلية العالية على امتزاز المواد كصبغة المثيلين

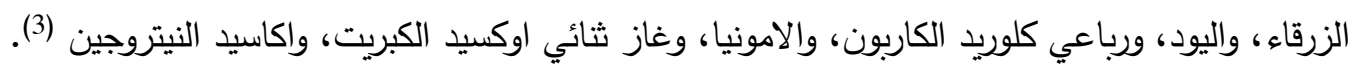

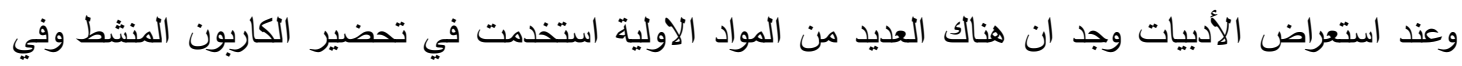
مايلي بعضا منها: تمكن الغنام(4) وجماعته من تحضير كاربون منشط من اشجار التوت باستخدام نسب مختلفة من

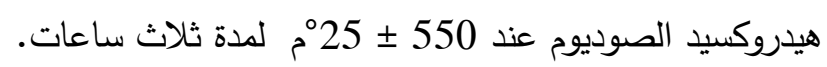

كما تمكن عويد(5) من تحضير كاربون منشط من عدد من المخلفات النباتية وباستخدام كمية اضافية

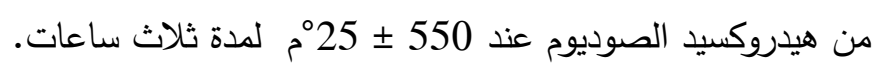

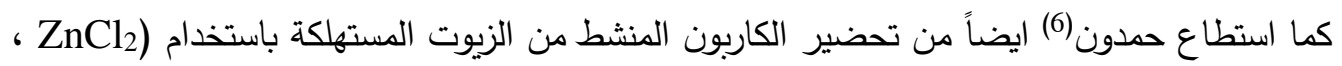

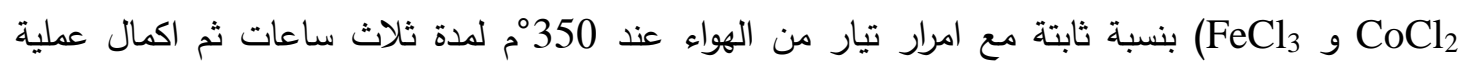

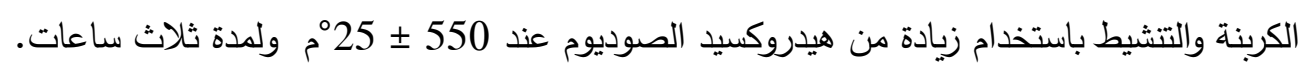

وحضر Ananthabaskaran

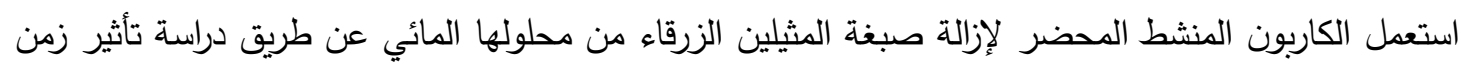
التماس وكمية المادة المازة وتركيز الصبغة والدالة الحامضية ودرجة الحرارة. ان الكاربون المنشط بالإمكان استعماله استعمالات مختلفة منها: امتزاز الابخرة والغازات وفي ازالة وداتة

الالوان وتتقية السوائل فضلا عن استخدامه كمادة ساندة للحفازات(9,8).

Materials and methods

Chemicals Materials

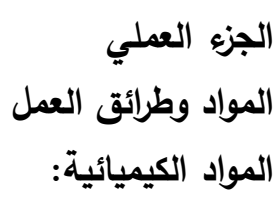

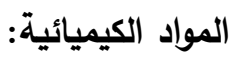

\begin{tabular}{|c|c|}
\hline \multicolumn{2}{|c|}{ المواد الكيميائية } \\
\hline Hydrochloric acid & حامض الهيدروكلوريك \\
\hline Acetone & اسيتون \\
\hline Sodium hydroxide & هيدروكسيد الصوديوم \\
\hline Iodine & يود \\
\hline \multirow[t]{2}{*}{ Zinc chloride } & كلوريد الخارصين \\
\hline & دليل زهرة الشمس \\
\hline Potassium iodide & يوديد البوتاسيوم \\
\hline Sodium thio sulphate & ثايوسلفات الصوديوم \\
\hline Starch & 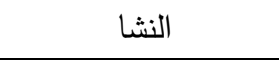 \\
\hline Methylene Bule & صبغة المثيلين الزرقاء \\
\hline
\end{tabular}

جدول (1): المواد الكيميائية المستخدمة

الكاربون 
تم في بحثا هذا تحضير الفحم المنشط من مخلفات بذور نبات القريص بعد نقعها بالأسيتون مدة (3)

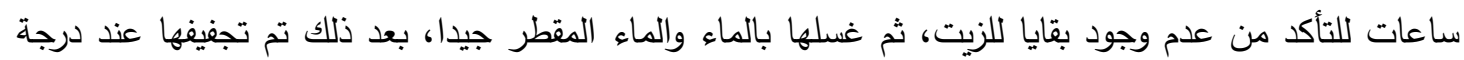
(100مْ) مدة (4) ساعات، ثم بعد ذلك تم سحق مخلفات البذور ومن ثم تمت كربنتها كربنة اولية بدرجة

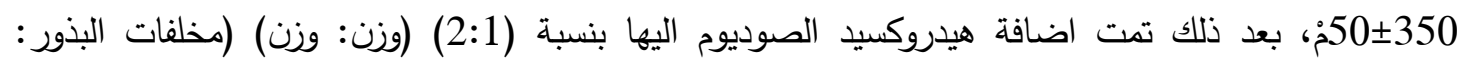

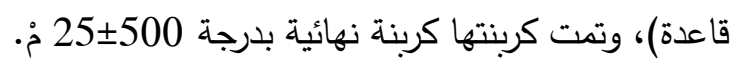

\section{Study of proparties at activated carbon}

دراسة صفات الكاربون المنشط المحضر تم تحديد صفات الكاربون المنشط عن طريق تحديد قابلية امتزاز اليود من محلوله المائي(10)، وامتزاز صبغة المثلين الزرقاء من محلولها المائي(11)، فضلا عن قياس كل من من محتوى الرماد (12)،هحتوى الرطوبة (10) والكثافة(13).

\section{Results and discussion}

النتائج والمناقشة

Choice of the Plant Sample for Study اختيار انموذج النبات قيد الدراسة: استعمل في تحضير الكاربون المنشط في بحثنا هذا مخلفات بذور نبات القريص ذي الاسم العلمي لUrtica dioica الخصبة وجوانب الطرق في مناطق مختلفة من محافظة نينوى، وبالقرب من منطقة الشيخان إذ يتوفر بكميات اكبر ، وقد تم عزل البذور وتتظيفها من الثوائب واستخلاص الزيوت منها التي استعملت كمادة اولية لانتاج وقود

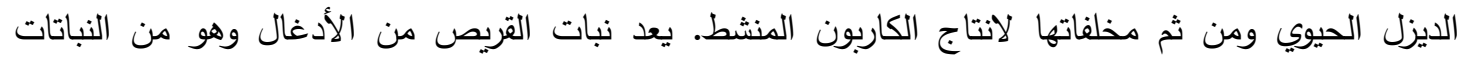
الحولية التي تظهر وتتموفي الرطوبة ابتداءً من شهر كانون الاول وتمتد الى أواخر شهر نيسان تقريبا. وتصنيف هذا النبات كالآتي (14):

جدول(2): تصنيف نبات القريص

\begin{tabular}{|c|c|}
\hline Kingdom & Plantae-Plants \\
\hline Subkingdom & Tracheobionta-vascular plants \\
\hline Superdivision & Spermatophyte-seed plants \\
\hline Division & Magnoliophta-Flowering plants \\
\hline Class & Magnoliopsida-Dicotyledons \\
\hline Order & Urticales \\
\hline Family & Urticaceae \\
\hline Genus & Urtica \\
\hline Species & dioica \\
\hline
\end{tabular}

جدول(3): الصفات الفيزيائية المقاسة والفعالية لإنموذج الكاربون المنشط المحضر بالتنشيط الحراري

\begin{tabular}{|c|c|c|c|c|c|}
\hline ملفزاز اليود & الزتزاز صبغة المثيلين & الرماد \% & الرطوبة \% & الكثافة غم/سم3 & النموذج \\
\hline 143.9 & 24.00 & 1.75 & 0.511 & 0.34210 & النموذج المحضر \\
\hline 445.00 & 63.800 & 1.50 & 0.530 & 0.35600 & B.D.H ${ }^{(15)}$ \\
\hline 908.00 & 90.0 & 3.90 & 0.620 & 0.32500 & Russ. ${ }^{(15)}$ \\
\hline
\end{tabular}

جدول(4): الصفات الفيزيائية المقاسة لإنموذج الكاربون المنشط المحضر باستعمال زيادة من NaOH منات بنسبة (2:1) (وزن:وزن) (مخلفات بذور : قاعدة) 


\begin{tabular}{|c|c|c|c|c|c|}
\hline ملفف/غم اليود & امتزاز صبغة المثيلين & الرماد \% & الرطوبة \% & غم/سم3 & النموذج \\
\hline 483.00 & 65.00 & 1.6 & 0.520 & 0.34770 & النموذج المحضر \\
\hline
\end{tabular}

نلاحظ من الجداول أعلاه ان صفات الكاربون المنشط تغيرت الى الأفضل عند استعمال قاعدة، وهذا

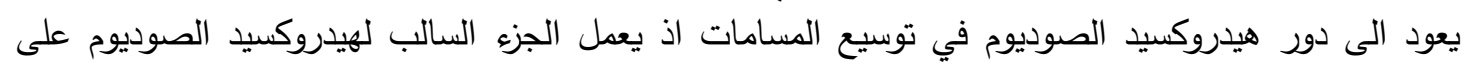

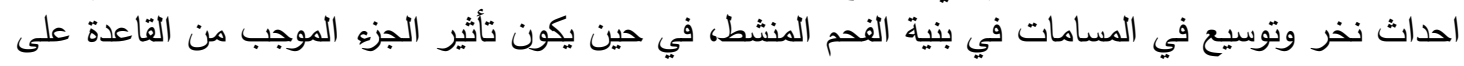

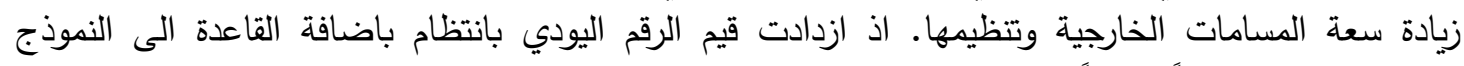
الاصلي ونلاحظ ايضاً ازدياداً في تركيز صبغة المتانة المثلين الزرقاء الممتزة.

الاستنتاجات

1- يمكن اعتبار مخلفات بذور نبات القربص مادة أولية لتحضير الكاربون المنشط.

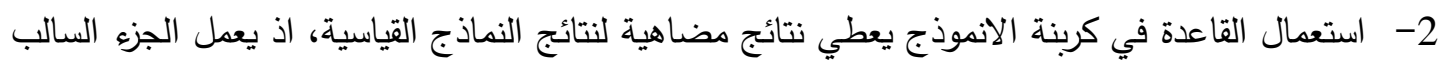

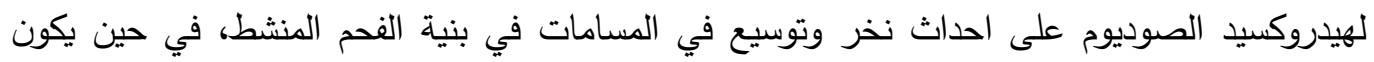

تأثير الجزء الموجب من القاعدة على زيادة سعة المسامات الخارجية وتتظيمها.

\section{References}

(المصادر

1) Duong D. Do. (1998), Adsorption Analysis: Equilibria and Kinetics, British Library Cataloguing-in-Publication Data Copyright 1998 by Imperial College Press.

2) O.M. Ramadhan \& M.A. Rigibi, (2000), "Activated Carbon by modified Carbonization", Vol .46. Scince and education .

3) P.L. Hart \& P.L. Walker, Jr. (1967), "Oxygen Chemisorption on Awell Cleaned Carbon Surface", Carbon, Vol . 5, pp.363-366.

4) Abdallah KH.A., Awaid KH.A., Hamdoon A.A.,"preparation of Activated carbon...", almajala alqtria,Volume 13,pp. 26-33. 2004.(In Arabic).

5) K.A. Ewaid, "Production of Activated Carbon From So Chemistry Agricultural Wastes by Chemical Treatment" National Journal of, 2005, Volume 17, pp.138142.

6) A.A. Hamdon, 2005, "Production of Activated Carbon From Spent Lubricoling Oils by Chemical Treatment", National Journal of Chemistry Vol.(18), 216-225.

7) Ananthabaskaran T. and et al, (2011), "Adsorptive Removal of Methylene Blue onto $\mathrm{ZnCl}_{2}$ Activated Carbon from Wood Apple Outer Shell: Kinetics and Equilibrium Studies", E-Journal of Chemistry, 2011, 8(4), 1696-1707.

8) J.G. King, (1976), "Fuel solid, liquid \& Gaseous", Ltd., London, $6^{\text {th }}$ Ed., p40.

9) S.N. Ahmed, J.M. Stencel, F.J. Derby, \& R.M. Baldwin, (1993), "Activated Carbon for the removal of Nitric-Oxide", J.Fuel processing techhnology, Vol. 34, Jss. 29 pp. 123-136.

10) ISO, 5.62, (1981), "Determination of Volatile Matter Content of Hard Coal \& KoKe", The Full Text Can be Obtained from ISO Central.

11) ASTM D2854, 70 Standard Test Method for Apparent Density of Activated carbon.

12) "Test Method for Activated Carbon", Roster bau int. Engineering GMBH, W.Germany Devtschos Arzneibuch $6^{\text {th }}$ Edition .

13) B.V. Baharudin, \& W.K. Hoi, (1987), "The Quality of charcoal from Various types of wood", Fuel, Vol. 66., p.1305.

14) Almorfaq al alami., Bioinformition.,"Urtica dioica", (2011).

15) F.F.Saleem, (1997), Production of Activated Carbon from local Raw materials/Effect of structural modification on physical and mechanical properties, Ph.D. thesis, university of Mosul. 